\title{
Use of endoscopic nasobiliary drainage tube for treating mediastinitis caused by insertion of an esophageal self-expanding metal stent
}

Self-expanding metal stents (SEMS) are relatively easy to deploy, with a high technical success rate, and provide rapid relief of dysphagia [1], which is the major factor affecting quality of life in patients not suitable for resection. Since Domschke et al. first reported the use of SEMS in 1990 [2], the technique has been widely used for malignant esophageal stenosis [3], although there have been many reports of complications associated with the combination of SEMS and chemoradiation therapy (CRT) [4,5].

A 56-year-old man was diagnosed as having carcinoma of the lower esophagus in July 2008. After CRT, he was admitted to our hospital for second-line chemotherapy in October 2008. The patient had developed uncontrollable salivation in the month prior to admission. A SEMS was placed successfully, but he developed fever and low blood pressure on November 28,2008 . Computed tomography (CT) examination revealed a fistula between the esophagus at the oral end of the SEMS and the mediastinum, and mediastinitis was suspected. Despite the administration of antibiotics, and steroid pulse and $\gamma$-globulin combination therapy, an abscess with liquefaction was confirmed in the mediastinum 7 days later ( $\bullet$ Fig. 1 ). An endoscopic nasobiliary drainage (ENBD) tube was placed in the mediastinal abscess along a guide wire to allow drainage ( $\bullet$ Fig. 2).

A CT scan demonstrated improvement in the abscess 12 days after insertion of the ENBD tube ( $\bullet$ Fig. 3 ).
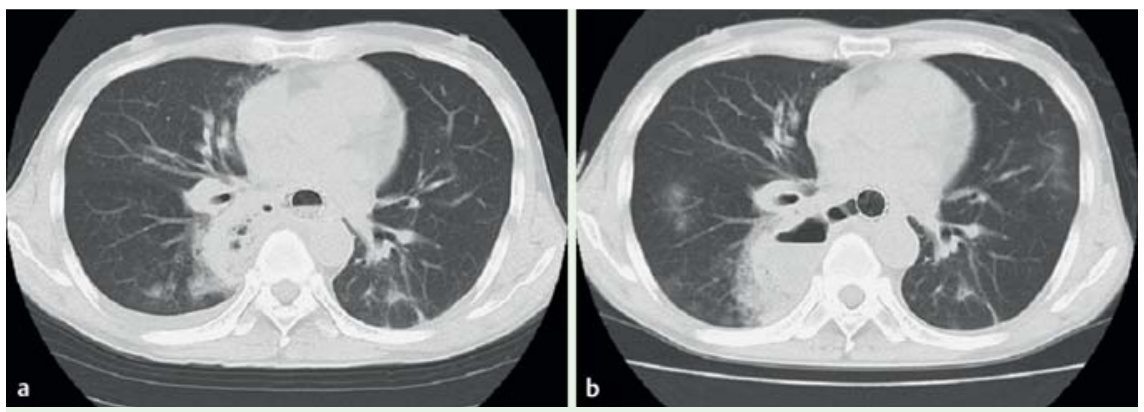

Fig. 1 CT examination after first SEMS insertion. a There is an infiltrative shadow between the mediastinum and the esophagus. Consolidation on the dorsal side suggests air inclusion. Formation of a fistula between the esophagus and the mediastinum was suspected. Septic shock caused by mediastinitis was diagnosed. $\mathbf{b}$ An abscess with liquefaction in the mediastinum, noted 7 days later despite the administration of antibiotics, and steroid pulse and $y$-globulin combination therapy.
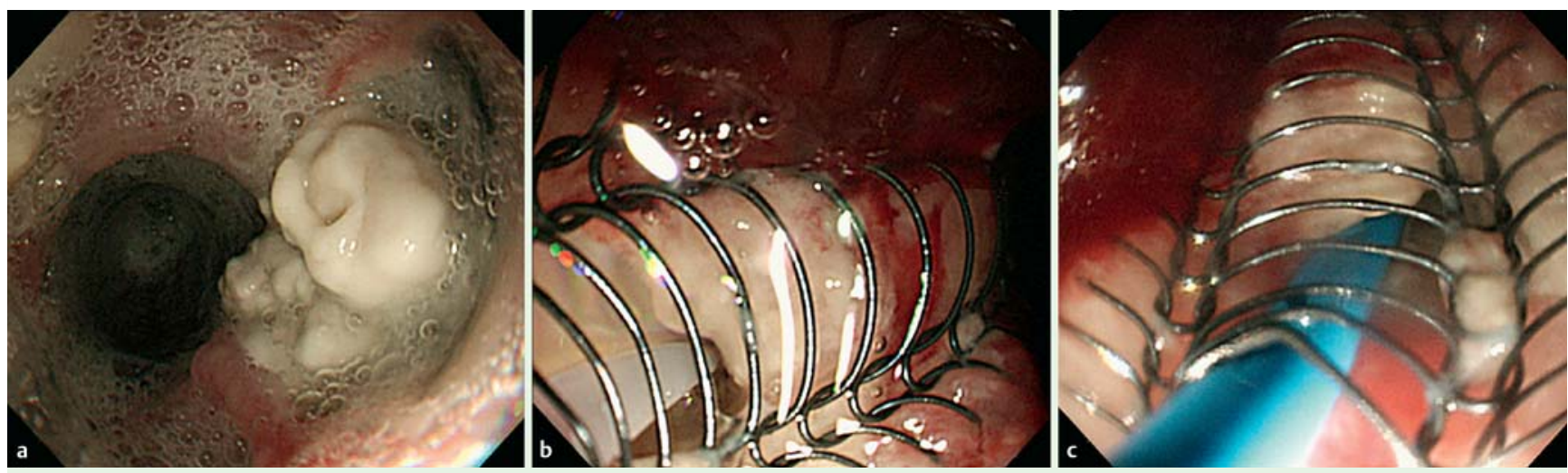

Fig. 2 Upper gastrointestinal endoscopy. a Partial granulation accompanied by white necrotic tissue at the oral edge of the stent. $\mathbf{b}$ Removal of the granulation tissue revealing the fistula between the esophagus and the mediastinum. An endoscopic retrograde cholangiopancreatography (ERCP) catheter was inserted into the fistula. c A 6-Fr endoscopic nasobiliary drainage (ENBD) tube was placed in the mediastinal abscess along the guide wire. 


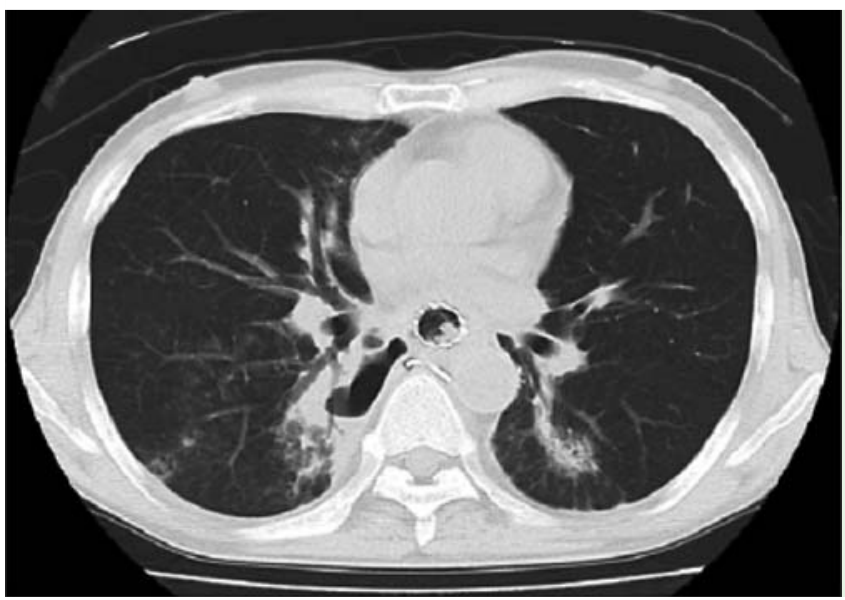

Fig. 3 Radiological follow-up after endoscopic nasobiliary drainage (ENBD) tube drainage. Computed tomography (CT) examination confirmed adequate drainage of the mediastinal abscess, and revealed improvement of the abscess 12 days after tube insertion.

K. Soga ${ }^{1,4}$, J. Ochiai ${ }^{2,4}$, T. Miyajima ${ }^{3,4}$, Y. Naito ${ }^{4}$, T. Yoshikawa ${ }^{4}$

1 Department of Internal Medicine, Nishijin Hospital, Kyoto, Japan

2 Department of Gastroenterology, Gakkentoshi Hospital, Kyoto, Japan

3 Department of Gastroenterology, Uji Takeda Hospital, Kyoto, Japan

4 Department of Molecular Gastroenterology and Hepatology, Kyoto Prefectural University of Medicine, Kyoto, Japan

\section{References}

1 Dua KS. Stents for palliating malignant dysphagia and fistula: is the paradigm shifting? Gastrointest Endosc 2007; 65: 77-81

2 Domschke W, Foerster EC, Matek Wet al. Selfexpanding mesh stent for esophageal cancer stenosis. Endoscopy 1990; 22: 134-136

3 Raijman I, Siddique I, Ajani J et al. Palliation of malignant dysphagia and fistulae with coated expandable metal stents: experience with 101 patients. Gastrointest Endosc 1998; 48: $172-179$
4 Kinsman KJ, DeGregorio BT, Katon RM et al. Prior radiation and chemotherapy increase the risk of life-threatening complications after insertion of metallic stents for esophagogastric malignancy. Gastrointest Endosc 1996; 43: 196-203

5 Muto M, Ohtsu A, Miyata Yet al. Self-expandable metallic stents for patients with recurrent esophageal carcinoma after failure of primary chemoradiotherapy. Jpn J Clin Oncol 2001; 31: $270-274$

Bibliography

DOI $10.1055 / \mathrm{s}-0030-1256287$

Endoscopy 2011; 43: E223 -E224

(c) Georg Thieme Verlag KG Stuttgart . New York . ISSN 0013-726X

\section{Corresponding author}

K. Soga

Nishijin Hospital

Department of Internal Medicine

1035 Mizomae-cho

Kamigyo-ku

Kyoto 602-8319

Japan

Fax: +81-75-461-5514

sogatti@koto.kpu-m.ac.jp 